\title{
Correction to: The Association Between Obesity and Hyperactivity/Anxiety Among Elementary School Students in Japan
}

\author{
Yohei Suzuki $^{1} \cdot$ Ai lkeda $^{1} \cdot$ Koutatsu Maruyama $^{2} \cdot$ Naoko Sakamoto $^{3} \cdot$ Hiroo Wada $^{1} \cdot$ Takeshi Tanigawa $^{1}$ \\ Published online: 21 January 2020 \\ (C) International Society of Behavioral Medicine 2020
}

\section{Correction to: International Journal of Behavioral Medicine} https://doi.org/10.1007/s12529-019-09827-x

The original article has been corrected:

One of the formulas was incorrect, and the corrected formula is shown below:

RERI $=\operatorname{exponent}(\beta 1+\beta 2+\beta 3)-\operatorname{exponent}(\beta 1)-\operatorname{exponent}(\beta 2)+1$

The online version of the original article can be found at https://doi.org/ 10.1007/s12529-019-09827-x

\section{Takeshi Tanigawa}

tataniga@juntendo.ac.jp

1 Department of Public Health, Graduate School of Medicine, Juntendo University, 2-1-1 Hongo, Bunkyo-ku, Tokyo, Japan

2 Department of Bioscience, Graduate School of Agriculture, Ehime University, Matsuyama, Ehime, Japan

3 Department of Epidemiologic Research, Toho University, Tokyo, Japan 